Nr. $168 / 93$

On a Dual Pair of Spaces of Smooth and

Generalized Random Variables

J. Potthoff and M. Timpel 


\title{
On a Dual Pair of Spaces of Smooth and Generalized Random Variables
}

\author{
Jürgen Potthoff and Matthias Timpel \\ Universität Mannheim, Mannheim
}

\begin{abstract}
A dual pair $\mathcal{G}$ and $\mathcal{G}^{*}$ of smooth and generalized random variables, respectively, over the white noise probability space is studied. $\mathcal{G}$ is constructed by norms involving exponentials of the Ornstein-Uhlenbeck operator, $\mathcal{G}^{*}$ is its dual. Sufficient criteria are proved for when a function on $\mathcal{S}(\mathbb{R})$ is the $\mathcal{S}$-transform of an element in $\mathcal{G}$ or $\mathcal{G}^{*}$.
\end{abstract}

\section{Introduction}

Dual pairs of spaces of random variables have been studied and applied to many problems of stochastic and infinite dimensional analysis in numerous papers. We refer the reader to [HKP 93] for the background of the subject and for an extensive list of references. The two most used pairs of spaces seem to be the Hida spaces $\left(\mathcal{S}^{(*)}\right)$ and the Meyer-Watanabe spaces $\mathcal{D}^{(*)}$.

In [PS 91] a result was proved for the space $(\mathcal{S})^{*}$ of Hida distributions which characterizes this space in terms of its $S$-transform, essentially a Gauß-Laplace transform. This result appears to be quite useful, as well from a structural point of view as for applications. For example, this "characterization theorem" has been applied to: quantum field theory [PS 93], Feynman integrals [FPS 91, KS 92, LLS 93], (anticipating) stochastic (Volterra, partial) differential equations [CLP 93, CP 92, KP 90, Po 92, Po 93], law of large numbers and central limit theorem of Donsker's delta functional [PS 93ab]. On the other hand, for applications in stochastic analysis the pair $\mathcal{D}$ and $\mathcal{D}^{*}$ of Meyer-Watanabe seems to be more fitting: for example, the solutions of non-degenerate Itô equations belong to $\mathcal{D}$ [Wa 84] and not to $(\mathcal{S})$. This is due to the fact that elements in $(\mathcal{S})$ have a chaos decomposition with kernels in $\mathcal{S}\left(\mathbb{R}^{n}\right)$ while solutions of SDE's have kernels which fail to be in $\mathcal{S}\left(\mathbb{R}^{n}\right)$. Unfortunately, there is no characterization-type theorem known for the pair $\left(\mathcal{D}^{*}, \mathcal{D}\right)$, and such a characterization appears to be a rather difficult problem. Therefore the basic idea of the present paper is to consider a space of random variables bigger than $(\mathcal{S})$ but smaller than $\mathcal{D}$ which would possibly contain solutions of Itô SDE's (in particular having kernels of the chaos decomposition in $L^{2}\left(\mathbb{R}^{n}\right)$ ) but which at the same time allows for a characterization in terms of the $\mathcal{S}$-transform. 
In fact, there is a pair denoted by $\left(\mathcal{G}, \mathcal{G}^{*}\right)$ which is "between" the above mentioned pairs, namely we have the embeddings

$$
(\mathcal{S})^{*} \supset \mathcal{G}^{*} \supset \mathcal{D}^{*} \supset\left(L^{2}\right) \supset \mathcal{D} \supset \mathcal{G} \supset(\mathcal{S})
$$

Although we could not find an equivalent characterization of $\mathcal{G}^{*}$ and $\mathcal{G}$ in terms of their $S$-transforms, we prove in Section 4 a sufficient condition for functions on $\mathcal{S}(\mathbb{R})$ to be the $\mathcal{S}$-transform of an element in $\mathcal{G}$ or in $\mathcal{G}^{*}$. On the other hand, $\mathcal{G}$ is large enough to contain at least some solutions of Itô-equations: indeed, we show in Section 2 that the solution of the SDE defining the stochastic exponential of Brownian motion belongs to $\mathcal{G}$. Thus there is some hope that this dual pair is appropriate for the study of stochastic differential equations, and that for this the power of characterization-type results becomes available.

The space $\mathcal{G}$ is constructed by $L^{2}$-norms with exponential weights of the OrnsteinUhlenbeck operator. It has been considered in several papers, e.g. [LM 88, ÜZ 93].

The organization of this paper is as follows. In Section 2 we define $\mathcal{G}$ and $\mathcal{G}^{*}$ and prove some of their elementary properties. In particular we show that $\mathcal{G}$ is a Fréchet algebra. In Section 3 we show some properties concerning the calculus on $\mathcal{G}$. For example, we prove the convergence of the Taylor series (in the sense of convergence in $\mathcal{G}$ ) for translations in the directions of $L^{2}(\mathbb{R})$. In Section 4 we prove our main result, namely the above mentioned criterium on ray-analytic functions on $\mathcal{S}(\mathbb{R})$ which allows to conclude that such a function $F$ is the $\mathcal{S}$-transform of an element in $\mathcal{G}$ or in $\mathcal{G}^{*}$. The main point is to find a condition on $F$ which implies that the kernels of $\mathcal{S}^{-1} F$ are in $L^{2}\left(\mathbb{R}^{n}\right)$. This can be achieved by asking for an exponential bound in terms of a trace class quadratic form on $\mathcal{S}(\mathbb{R})$ (see Section 4). We also prove a convergence result, discuss examples and the Wick product.

Acknowledgement. It is a pleasure to thank L. Streit for stimulating discussions.

\section{Definition of $\mathcal{G}, \mathcal{G}^{*}$, and Elementary Properties}

Throughout this paper we work with the white noise probability space $\left(\mathcal{S}^{\prime}(\mathbb{R}), \mathcal{B}, \mu\right)$ as the underlying probability space. $\left(L^{p}\right)$ is a shorthand for $L^{p}\left(\mathcal{S}^{\prime}(\mathbb{R}), \mathcal{B}, \mu\right), p \geq 1 . N$ denotes the number or Ornstein-Uhlenbeck operator, i.e., $N$ acts on the $n$-th homogeneous chaos of $\left(L^{2}\right)$ as multiplication by $n, n \in N_{0}$. By $\mathcal{P}$ we denote the algebra of polynomials which is dense in $\left(L^{2}\right): \mathcal{P}$ is generated by the canonical coordinate process $\xi \mapsto X_{\xi}, X_{\xi}(\omega)=$ $<\omega ; \xi>, \xi \in \mathcal{S}(\mathbb{R}), \omega \in \mathcal{S}^{\prime}(\mathbb{R})$.

Since every $\varphi \in \mathcal{P}$ has a chaos decomposition

$$
\varphi=\sum_{n=1}^{\infty} \varphi^{(n)}
$$

with only finitely many non-zero $\varphi^{(n)}, n \in N_{0}$, we have $e^{\lambda N} \varphi \in\left(L^{2}\right)$ for every $\lambda \in \mathbb{R}$. Thus we may define a family $\left\{\|\cdot\|_{\lambda}, \lambda \in \mathbb{R}\right\}$ of norms $\|\cdot\|_{\lambda}$ on $\mathcal{P}$ by setting

$$
\|\varphi\|_{\lambda}:=\left\|e^{\lambda N} \varphi\right\|_{\left(L^{2}\right)}
$$


Note that $\varphi^{(n)}=I_{n}\left(f^{(n)}\right)$ where $I_{n}\left(f^{(n)}\right)$ is the $n$-fold Wiener integral of $f^{(n)} \in L^{2}\left(\mathbb{R}^{n}\right)$ (in fact, here $f^{(n)} \in \mathcal{S}\left(\mathbb{R}^{n}\right)$ ). Thus we have

$$
\|\varphi\|_{\lambda}=\left(\sum_{n=0}^{\infty} n ! e^{2 \lambda n}\left\|f^{(n)}\right\|_{L^{2}\left(\mathbb{R}^{n}\right)}^{2}\right)^{\frac{1}{2}} .
$$

Now we define $\mathcal{G}_{\lambda}, \lambda \in \mathbb{R}$, as the completion of $\mathcal{P}$ with respect to $\|\cdot\|_{\lambda}$. Thus $\mathcal{G}_{\lambda}$ is in one-toone correspondence with the (Fock type) space consisting of all sequences $\left\{f^{(n)}, n \in N_{0}\right\}$ of symmetric elements $f^{(n)}$ in $L^{2}\left(\mathbb{R}^{n}\right), n \in \mathbb{N}, f^{(0)} \in \mathbb{C}$, so that the sum in (2.3) converges.

It is clear that for $\lambda^{\prime} \leq \lambda$, we have $\mathcal{G}_{\lambda} \subseteq \mathcal{G}_{\lambda^{\prime}}$. Now we set

$$
\mathcal{G}:=\bigcap_{\lambda \in \mathbb{R}} \mathcal{G}_{\lambda}
$$

and equip $\mathcal{G}$ with the projective limit topology. It is obvious that we can replace the intersection over $\lambda \in \mathbb{R}$ in (2.4) by the intersection over $\lambda \in \mathbb{N}$. Since the norm (2.2) is Hilbertian, $\mathcal{G}$ is a countably Hilbert space (e.g., [GV 68]), and hence metrizable. Moreover, the fact that $e^{\lambda N}, \lambda \in \mathbb{R}$, is closed on $\left(L^{2}\right)$ entails that the family $\left\{\|\cdot\|_{\lambda}, \lambda \in \mathbb{R}\right\}$ is compatible in the sense of [GV 68]. Therefore $\mathcal{G}$ is complete [GV 68]. Finally note that as a countably Hilbert space $\mathcal{G}$ is reflexive (e.g., [GV 68]). Thus $\mathcal{G}$ is a reflexive Fréchet space.

By definition, $\mathcal{G}^{*}$ is the dual of $\mathcal{G}$. We have

$$
\mathcal{G}^{*}=\bigcup_{\lambda \in \mathbb{R}} \mathcal{G}_{\lambda}
$$

Example 2.1. Consider the Brownian motion $\left\{B(t)=X_{1_{[0, t)}} ; t \geq 0\right\}$, where $X$. denotes also the extension of the canonical coordinate process from $\mathcal{S}(\mathbb{R})$ to $L^{2}(\mathbb{R})$. Let $Y(t)$ be the solution of

$$
\begin{aligned}
d Y(t) & =Y(t) d B(t), \quad t>0 \\
Y(0) & =1
\end{aligned}
$$

i.e., $Y(t)=\exp \left(B(t)-\frac{1}{2} t\right)$. Then $Y(t)$ has a chaos decomposition which is given by the sequence of kernels $\left\{f^{(n)}=\frac{1}{n !} 1_{[0, t)}^{\otimes n}, n \in \mathbb{N}_{0}\right\}$ (with the convention $0 !=1$ ). Let $\lambda \in \mathbb{R}$, then

$$
\begin{aligned}
\|Y(t)\|_{\lambda}^{2} & =\sum_{n=0}^{\infty} n ! \frac{1}{n !^{2}} e^{2 \lambda n} t^{n} \\
& =\exp \left(e^{2 \lambda} t\right) .
\end{aligned}
$$

Thus we have $Y(t) \in \mathcal{G}$ for all $t \geq 0$. (A somewhat slicker proof can be done by using the facts that $\exp (\lambda N)$ is equal to the second quantization $\Gamma\left(e^{\lambda}\right)$ of $e^{\lambda}$, considered as a multiplication operator on $L^{2}(\mathbb{R})$, and that for a closed operator $B$ on $L^{2}(\mathbb{R})$ and $f \in \mathcal{D}(B)$ we have

$$
\Gamma(B): e^{X_{f}}:=: e^{X_{B f}}:
$$


where $: \exp X_{f}:=\exp \left(X_{f}-\frac{1}{2}\|f\|_{L^{2}(\mathbb{R})}^{2}\right)$. Then we have immediately

$$
\begin{aligned}
e^{\lambda N} Y(t) & =: e^{e^{\lambda} B(t)}: \\
& =e^{e^{\lambda} B(t)-\frac{1}{2} e^{2 \lambda_{t}} t}
\end{aligned}
$$

and the last expression has $\left(L^{2}\right)$-norm equal to $\exp \left(\frac{1}{2} e^{2 \lambda} t\right)$.)

Example 2.2. Let $x \in \mathbb{R}, t>0$, and consider Donsker's delta function $\delta_{x} \circ B(t)$. It has a well-known chaos decomposition $\left(\right.$ in $\left.(\mathcal{S})^{*}\right)$ given by (e.g., [HKP 93])

$$
\delta_{x} \circ B(t)=p(t, x) \sum_{n=0}^{\infty} \frac{1}{n !} t^{-n} H_{n, t}(x) H_{n, t} \circ B(t),
$$

where $p(t, x)=(2 \pi t)^{-\frac{1}{2}} \exp \left(-\frac{1}{2 t} x^{2}\right)$, and $H_{n, t}$ is the $n$-th Hermite polynomial with variance $t$ :

$$
H_{n, t}(x)=\left.\frac{d^{n}}{d s^{n}} \exp \left(s x-\frac{1}{2} s^{2} t\right)\right|_{s=0} .
$$

Now write

$$
\delta_{x} \circ B(t)=p(t, x)^{\frac{1}{2}} \sum_{n=0}^{\infty} \frac{1}{\sqrt{n ! t^{n}}} e_{n, t}(x) H_{n, t} \circ B(t),
$$

with the Hermite function

$$
e_{n, t}(x)=\frac{1}{\sqrt{n ! t^{n}}} p(t, x)^{\frac{1}{2}} H_{n, t}(x) .
$$

Let $\lambda>0$. Then

$$
\left\|\delta_{x} \circ B(t)\right\|_{-\lambda}^{2}=p(t, x) \sum_{n=0}^{\infty} e^{-2 \lambda n_{e}} e_{n, t}(x)^{2} .
$$

By a standard estimate on Hermite functions [HP 57, (21.3.2)] we have $e_{n, t}(x)=0\left(n^{-\frac{1}{4}}\right)$. Thus the sum converges for any $\lambda>0$, and we have $\delta_{x} \circ B(t) \in \mathcal{G}_{-\lambda}, \lambda>0$, and in particular $\delta_{x} \circ B(t) \in \mathcal{G}^{*}$.

Examples 2.1 and 2.2 will be reconsidered in Section 4 .

Next we establish the inclusion relations (1.1). First we need some more notation. By $\|\cdot\|_{p, k}$ we denote the norm on $\mathcal{P}$ which is given by

$$
\|\varphi\|_{p, k}:=\left\|(1+N)^{\frac{k}{2}} \varphi\right\|_{\left(L^{p}\right)},
$$

where $k \in \mathbb{Z}, p \geq 1 . \mathcal{D}^{p, k}$ is the completion of $\mathcal{P}$ with respect to $\|\cdot\|_{p, k}$, and the MeyerWatanabe space $\mathcal{D}$ is the projective limit of the family $\left\{\mathcal{D}^{p, k} ; p \geq 1, k \in \mathbb{N}\right\}$ (cf., e.g., [FP $89, \mathrm{Me} \mathrm{83}, \mathrm{Wa} 83, \mathrm{Su} 85$ ], or in the white noise context [HKP 93]). $\mathcal{D}^{*}$ is the dual of $\mathcal{D}$.

Let $A$ be the self-adjoint operator on $L^{2}(\mathbb{R})$ which is given on $\mathcal{S}(\mathbb{R})$ by:

$$
(A \xi)(x)=-\xi^{\prime \prime}(x)+\left(1+x^{2}\right) \xi(x), \quad \xi \in \mathcal{S}(\mathbb{R}), x \in \mathbb{R} .
$$


$\Gamma(A)$ denotes its second quantization (e.g., [Si 74, HKP 93]) which is a self-adjoint operator on $\left(L^{2}\right)$. Since infspec $A=2$, it is not hard to see that infspec $\Gamma(A)=1$ (with 1 being the normalized eigenvector in $\left(L^{2}\right)$ of $\Gamma(A)$ of eigenvalue 1$)$. We define the norms

$$
\|\varphi\|_{2, p}:=\left\|\Gamma(A)^{p} \varphi\right\|_{\left(L^{2}\right)}, \quad p \in \mathbb{R},
$$

on $\mathcal{P}$, and denote the completion of $\mathcal{P}$ with respect to $\|\cdot\|_{2, p}$ by $(\mathcal{S})_{p} \cdot(\mathcal{S})$ is the projective limit of the family $\left\{(\mathcal{S})_{p}, p \in \mathbb{R}\right\}$. The space $(\mathcal{S})^{*}$ of Hida distributions is the dual of $(\mathcal{S})$. For more details on these two spaces we refer the interested reader to [HKP 93, PS 91], and the references cited there.

Let us now show that $\mathcal{G} \subset \mathcal{D}$. Assume that $\varphi \in \mathcal{P}$, and let $k \in \mathbb{N}, p \geq 1$. Then we may estimate for large enough $\lambda \geq 0$ as follows:

$$
\begin{aligned}
\|\varphi\|_{\boldsymbol{p}, k} & =\left\|e^{-\lambda N}(1+N)^{\frac{k}{2}} e^{\lambda N} \varphi\right\|_{\left(L^{p}\right)} \\
& \leq\left\|e^{-\frac{\lambda}{2} N}(1+N)^{\frac{k}{2}} e^{\lambda N} \varphi\right\|_{\left(L^{2}\right)} \\
& \leq C_{\lambda, k}\left\|e^{\lambda N} \varphi\right\|_{\left(L^{2}\right)} \\
& =C_{\lambda, k}\|\varphi\|_{\lambda} .
\end{aligned}
$$

In the first estimate we used for large enough $\lambda$ (depending on $p$ ) Nelson's hypercontractivity theorem [Ne 73]. In the second we used the spectral theorem. Since $\mathcal{P}$ is dense in $\mathcal{G}$, the above estimate extends to $\varphi \in \mathcal{G}$.

Now let $p \geq 0$, and consider again $\varphi \in \mathcal{P}$. The fact that $\operatorname{infspec} A \geq 2$ entails that $\Gamma(A)^{-1} \leq 2^{-N}$, and therefore for given $\lambda \geq 0$ we can find $p \geq 0$ so that $e^{\lambda N} \Gamma(A)^{-p}$ is a contraction on $\left(L^{2}\right)$. Thus we have

$$
\begin{aligned}
\|\varphi\|_{\lambda} & =\left\|e^{\lambda N} \Gamma(A)^{-p} \Gamma(A)^{p} \varphi\right\|_{\left(L^{2}\right)} \\
& \leq\left\|\Gamma(A)^{p} \varphi\right\|_{\left(L^{2}\right)} \\
& =\|\varphi\|_{2, p} .
\end{aligned}
$$

Since $\mathcal{P}$ is dense in $(\mathcal{S})$, the preceding inequality holds for $\varphi \in(\mathcal{S})$. Altogether we have proved the following result (henceforth we identify $\left(L^{2}\right)$ with its dual):

Theorem 2.3. For the spaces $\mathcal{D}, \mathcal{G},(\mathcal{S})$ the following chain of continuous dense embeddings holds:

$$
\mathcal{D} \supset \mathcal{G} \supset(\mathcal{S})
$$

Consequently the chain of embeddings (1.1) holds.

In view of Theorem 2.3, Example 2.2 is rather trivial since Watanabe showed in [WA 83] that $\delta_{x} \circ X$ belongs to $\mathcal{D}^{*}$, and hence to $\mathcal{G}^{*}$, whenever $X$ is in $\mathcal{D}$ with non-degenerate Malliavin covariance matrix - which is clearly true for $B(t), t>0$.

We now show that $\mathcal{G}$ is an algebra under pointwise multiplication. This result has already been proved in [LM 88] with a quite different machinery than the one below. Our proof follows the one in [PY 92] of the corresponding statement for $(\mathcal{S})$ quite closely. 
Proposition 2.4. Let $\lambda_{0}:=\frac{1}{2} \ln (2+\sqrt{2})$ and assume that $\lambda>\lambda_{0}, \varphi, \psi \in \mathcal{G}_{\lambda}$. Then for all $\nu>\lambda_{0}, \varphi \cdot \psi \in \mathcal{G}_{\lambda-\nu}$ and there exists a constant $C_{\nu}$. so that

$$
\|\varphi \cdot \psi\|_{\lambda-\nu} \leq C_{\nu}\left\|_{\varphi}\right\|_{\lambda}\|\psi\|_{\lambda}
$$

Proof. Let $\varphi, \psi$ have the following chaos expansions

$$
\begin{aligned}
& \varphi=\sum_{n=0}^{\infty} I_{n}\left(f^{(n)}\right), \\
& \psi=\sum_{n=0}^{\infty} I_{n}\left(g^{(n)}\right),
\end{aligned}
$$

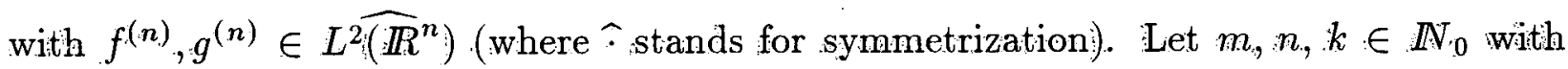
$k \leq m \wedge n$, set

$$
f^{(n)} \otimes_{k}, g^{(m)}=\int_{\mathbb{R}^{k}} f^{(n)}\left(\cdot, s_{1}, \ldots, s_{k}\right) g^{(m)}\left(\cdot, s_{1}, \ldots, s_{k}\right) d^{k} s
$$

and let $f^{(n)} \widehat{\otimes}_{k} g^{(m)}$ denote the symmetrization of $f^{(n)} \otimes_{k} g^{(m)}$. Then we have (e.g., [HKP 93, HPS 88])

$$
I_{n}\left(f^{(n)}\right) I_{m}\left(g^{(m)}\right)=\sum_{k=0}^{m \wedge n} k !\left(\begin{array}{c}
m \\
k
\end{array}\right)\left(\begin{array}{l}
n \\
k
\end{array}\right) I_{m+n-2 k}\left(f^{(n)} \widehat{\otimes}_{k} g^{(m)}\right)
$$

We may assume that $\nu \leq \lambda$, and estimate in the following way:

$$
\begin{aligned}
& \left\|I_{n}\left(f^{(n)}\right) I_{m}\left(g^{(m)}\right)\right\|_{\lambda-\nu} \leq \\
& \leq \sum_{k=0}^{m \wedge n} k !\left(\begin{array}{l}
m \\
k
\end{array}\right)\left(\begin{array}{l}
n \\
k
\end{array}\right) e^{(\lambda-\nu)(m+n-2 k)}\left\|I_{m+n-2 k}\left(f^{(n)} \widehat{\otimes}_{k} g^{(m)}\right)\right\|_{\left(L^{2}\right)} \\
& =\sum_{k=0}^{m \wedge n} k !\left(\begin{array}{l}
m \\
n
\end{array}\right)\left(\begin{array}{l}
n \\
k
\end{array}\right) e^{(\lambda-\nu)(m+n-2 k)}((m+n-2 k) !)^{\frac{1}{2}}\left\|f^{(n)} \widehat{\otimes}_{k} g^{(m)}\right\|_{L^{2}\left(\mathbb{R}^{n+m-2 k}\right)} \\
& \leq\left\|f^{(n)}\right\|_{L^{2}\left(\mathbb{R}^{n}\right): \| g^{(m)}} \|_{L^{2}\left(\mathbb{R}^{m}\right)} \sum_{k=0}^{m \wedge n} k !\left(\begin{array}{l}
m \\
k
\end{array}\right)\left(\begin{array}{l}
n \\
k
\end{array}\right)((m+n-2 k) !)^{\frac{1}{2}} e^{(\lambda-\nu)(m+n-2 k)} \\
& =\left\|I_{n}\left(f^{(n)}\right)\right\|_{\lambda} e^{-\nu n}\left\|I_{m}\left(g^{(m)}\right)\right\|_{\lambda} e^{-\nu m} \sum_{k=0}^{m \wedge n}\left[\left(\begin{array}{c}
m \\
k
\end{array}\right)\left(\begin{array}{l}
n \\
k
\end{array}\right)\left(\begin{array}{c}
m+n-2 k \\
n-k
\end{array}\right)\right]^{\frac{1}{2}} e^{-2 k(\lambda-i \nu)} \\
& \leq\left\|I_{n}\left(f^{(n)}\right)\right\|_{\lambda} e^{-\nu n}\left\|I_{m}\left(g^{(m)}\right)\right\|_{\lambda} e^{-\nu m} \sum_{k=0}^{m \wedge n}\left(\begin{array}{c}
m+n \\
2 k
\end{array}\right)^{\frac{1}{2}} \cdot 2^{\frac{1}{2}(m+n-2 k)} .
\end{aligned}
$$


Clearly we can bound the last sum by

$$
\begin{aligned}
2^{\frac{m+n}{2}} \sum_{k=0}^{m+n}\left(\begin{array}{c}
m+n \\
k
\end{array}\right)^{\frac{1}{2}} 2^{-\frac{k}{2}} & \leq 2^{\frac{m+n}{2}}\left(\sum_{k=0}^{\infty} 2^{-\frac{k}{2}}\right)^{\frac{1}{2}}\left(\sum_{k=0}^{m+n}\left(\begin{array}{c}
m+n \\
k
\end{array}\right) 2^{-\frac{k}{2}}\right)^{\frac{1}{2}} \\
& =\left(\frac{\sqrt{2}}{\sqrt{2}-1}\right)^{\frac{1}{2}}(\sqrt{2+\sqrt{2}})^{m+n}
\end{aligned}
$$

Therefore we get

$$
\left\|I_{n}\left(f^{(n)}\right) I_{m}\left(g^{(m)}\right)\right\|_{\lambda-\nu} \leq K \sigma^{n}\left\|I_{n}\left(f^{(n)}\right)\right\|_{\lambda} \sigma^{m}\left\|I_{m}\left(g^{(m)}\right)\right\|_{\lambda}
$$

where $K=\left(\frac{\sqrt{2}}{\sqrt{2}-1}\right)^{\frac{1}{2}}$ and $\sigma=\sqrt{2+\sqrt{2}} \cdot e^{-\nu}$. Now we estimate as follows

$$
\begin{aligned}
\|\varphi \cdot \psi\|_{\lambda-\nu} & =\left\|\sum_{m, n=0}^{\infty} I_{n}\left(f^{(n)}\right) I_{m}\left(g^{(m)}\right)\right\|_{\lambda-\nu} \\
& \leq \sum_{m, n=0}^{\infty}\left\|I_{n}\left(f^{(n)}\right) I_{m}\left(g^{(m)}\right)\right\|_{\lambda-\nu} \\
& \leq K\left(\sum_{n=0}^{\infty} \sigma^{n}\left\|I_{n}\left(f^{(n)}\right)\right\|_{\lambda}\right)\left(\sum_{n=0}^{\infty} \sigma^{n}\left\|I_{n}\left(g^{(n)}\right)\right\|_{\lambda}\right) \\
& \leq K \sum_{n=0}^{\infty} \sigma^{2 n}\left(\sum_{n=0}^{\infty}\left\|I_{n}\left(f^{(n)}\right)\right\|_{\lambda}^{2}\right)^{\frac{1}{2}}\left(\sum_{n=0}^{\infty}\left\|I_{n}\left(g^{(n)}\right)\right\|_{\lambda}^{2}\right)^{\frac{1}{2}} \\
& =K \frac{1}{1-\sigma^{2}}\|\varphi\|_{\lambda}\|\psi\|_{\lambda},
\end{aligned}
$$

if $\sigma<1$, i.e., if $\nu>\frac{1}{2} \ln (2+\sqrt{2})$.

An obvious consequence of Proposition 2.4 is the following

Theorem 2.5. Pointwise multiplication is continuous from $\mathcal{G} \times \mathcal{G}$ (equipped with the product topology) onto $\mathcal{G}$, and in particular $\mathcal{G}$ is an algebra with respect to pointwise multiplication.

We conclude this section by considering the Wick product, e.g., [MY 87, HKP 93, LOU 91] of elements in $\mathcal{G}^{*}$. Since $\mathcal{G}^{*} \subset(\mathcal{S})^{*}$, and since the Wick product $\Phi \diamond \Psi$ of elements $\Phi, \Psi$ in $(\mathcal{S})^{*}$ is an element in $(\mathcal{S})^{*}$ (cf., e.g., [HKP 93, Chapter 4]), $\Phi \diamond \Psi$ is also well-defined for $\Phi, \Psi \in \mathcal{G}^{*}$. $\Phi \diamond \Psi$ can be defined as follows: if $\Phi, \Psi$ have chaos decomposition given by $\left\{f^{(n)}, n \in \mathbb{N}_{0}\right\},\left\{g^{(n)}, n \in \mathbb{N}_{0}\right\}$, respectively, then $\Phi \diamond \Psi$ is determined by having the chaos decomposition given by $\left\{h^{(n)}, n \in N_{0}\right\}$, where

$$
h^{(n)}=\sum_{m=0}^{n} f^{(n-m)} \widehat{\otimes} g^{(m)} .
$$


In case that $\Phi, \Psi \in \mathcal{G}^{*}$, i.e., $\Phi, \Psi \in \mathcal{G}_{\lambda}$ for some $\lambda \in \mathbb{R}$, we have $f^{(n)}, g^{(n)} \in \widehat{L}^{2\left(\mathbb{R}^{n}\right)}, n \in$ $\mathbb{N}$. Let us denote from now on the norm of $L^{2}\left(\mathbb{R}^{n}\right)$ simply by $|\cdot| 2$ (there will be no danger of confusion). We have the estimate

$$
\left|h^{(n)}\right|_{2}^{2} \leq(n+1) \sum_{m=0}^{n}\left|f^{(n-m)}\right|_{2}^{2}\left|g^{(m)}\right|_{2}^{2}
$$

Let $\varepsilon>0$ and set $\lambda^{\prime}=\lambda-\varepsilon-\frac{1}{2}$, then we can estimate in the following way:

$$
\begin{aligned}
\|\Phi \diamond \Psi\|_{\lambda^{\prime}}^{2} & \leq \sum_{n=0}^{\infty}(n+1) ! e^{2 \lambda^{\prime} n} \sum_{m=0}^{n}\left|f^{(n-m)}\right|_{2}^{2}\left|g^{(m)}\right|_{2}^{2} \\
& =\sum_{n=0}^{\infty}(n+1) e^{2 \lambda^{\prime} n} \sum_{m=0}^{n}\left(\begin{array}{c}
n \\
m
\end{array}\right)\left\|\Phi^{(n-m)}\right\|_{2}^{2}\left\|\Psi^{(m)}\right\|_{2}^{2} \\
& \leq K_{\varepsilon}^{2} \sum_{n=0}^{\infty} \sum_{m=0}^{n} e^{2 \lambda(n-m)}\left\|\Phi^{(n-m)}\right\|_{2}^{2} e^{2 \lambda m}\left\|\Psi^{(m)}\right\|_{2}^{2} \\
& =K_{\varepsilon}^{2}\|\Phi\|_{\lambda}^{2}\|\Psi\|_{\lambda^{2}}^{2}
\end{aligned}
$$

Here we have denoted by $\Phi^{(n)}, \Psi^{(n)}$ the $n$-th multiple Wiener integral of $f^{(n)}, g^{(n)}$, respectively, and

$$
K_{\varepsilon}=(2 \varepsilon)^{-1 / 2} e^{-\left(\frac{1}{2}-\varepsilon\right)}
$$

Hence we have proved the following results:

Proposition 2.6. Let $\Phi, \Psi \in \mathcal{G}_{\lambda}, \lambda \in \mathbb{R}$. Let $\lambda_{0}=\lambda-\frac{1}{2}$, and $\lambda^{\prime}<\lambda_{0}$. Then $\Phi \diamond \Psi \in \mathcal{G}_{\lambda^{\prime}}$, and

$$
\|\Phi \diamond \Psi\|_{\lambda^{\prime}} \leq K_{\lambda, \lambda^{\prime}}\|\Phi\|_{\lambda}\|\Psi\|_{\lambda},
$$

where $K_{\lambda, \lambda^{\prime}}=\left(2\left(\lambda-\lambda^{\prime}\right)-1\right)^{-1 / 2} e^{\left(\lambda-\lambda^{\prime}\right)-1}$.

Theorem 2.7. $\mathcal{G}$ and $\mathcal{G}^{*}$ are algebras under the Wick product. Moreover, the Wick product is continuous from $\mathcal{G} \times \mathcal{G}$ (with product topology) onto $\mathcal{G}$.

\section{Calculus on $\mathcal{G}$}

In this section we give some basic results concerning the calculus on the spaces $\mathcal{G}_{\lambda}$. Some related results have also been mentioned in [ÜZ 93] (in the Wiener space setting). Most of the proofs are along standard lines (e.g., [HKP 93, PY 92]), and therefore will be somewhat sketchy.

For $h \in L^{2}(\mathbb{R})$ let $D_{h}$ denote the Gâteaux derivative in direction $h$. 
Proposition 3.1. Let $\lambda^{\prime}, \lambda \in \mathbb{R}, \lambda^{\prime}<\lambda, h \in L^{2}(\mathbb{R}), \varphi \in \mathcal{G}_{\lambda}$. Then $D_{h} \varphi \in \mathcal{G}_{\lambda^{\prime}}$ and the following estimate holds

$$
\left\|D_{h} \varphi\right\|_{\lambda^{\prime}} \leq C_{\lambda^{\prime}, \lambda}|h|_{2}\|\varphi\|_{\lambda}
$$

where $C_{\lambda^{\prime}, \lambda}=\left(2\left(\lambda-\lambda^{\prime}\right)\right)^{-1 / 2} e^{-\left(\lambda^{\prime}+1 / 2\right)}$.

Proof. It suffices to show the estimate (3.1) for $\varphi \in \mathcal{P}$. If $\varphi \in \mathcal{P}$ has a chaos expansion given by the sequence $\left\{f^{(n)}, n \in \mathbb{N}_{0}\right\}$ with $f^{(n)} \in L^{2}\left(\widehat{\mathbb{R}}^{n}\right), n \in \mathbb{N}, f^{(0)} \in \mathbb{C}(\odot$ stands for symmetrization), then $D_{h} \varphi$ has chaos expansion given by $\left\{(n+1)\left(h, f^{(n+1)}\right), n \in \mathbb{N}_{0}\right\}$, where

$$
\left(h, f^{(n+1)}\right)(u)=\int_{\mathbb{R}} h(s) f^{(n+1)}(s, u) d s, \quad u \in \mathbb{R}^{n} .
$$

Now inequality (3.1) follows easily from (2.3) and an application of Schwarz' inequality.

We conclude from Proposition 3.1 the following theorem:

Theorem 3.2. Let $h \in L^{2}(\mathbb{R}), \lambda \in \mathbb{R}, \varepsilon>0$. Then $D_{h}$ is continuous from $\mathcal{G}_{\lambda}$ into $\mathcal{G}_{\lambda-\varepsilon}$. In particular, $D_{h}$ is continuous on $\mathcal{G}$, and $\mathcal{G}$ is $C^{\infty}$ with respect to Gâteaux differentiation in all directions of $L^{2}(\mathbb{R})$.

Since $\mathcal{G}$ is an algebra with respect to pointwise multiplication (Theorem 2.5), it is clear that we have the usual product rule on $\mathcal{G}:$ for $\varphi, \psi \in \mathcal{G}$,

$$
D_{h}(\varphi \cdot \psi)=\left(D_{h} \varphi\right) \cdot \psi+\varphi \cdot\left(D_{h} \psi\right) .
$$

We remark that a corresponding rule holds also for the Wick product:

$$
D_{h}(\varphi \diamond \psi)=\left(D_{h} \varphi\right) \diamond \psi+\varphi \diamond\left(D_{h} \psi\right) .
$$

Firstly, all expressions make sense because of Theorem 2.7. Moreover, the kernels of the chaos decomposition of the left hand side of (3.2) are given by (cf. the arguments leading to Proposition 2.6)

$$
(n+1)\left(h, \sum_{m=0}^{n+1} f^{(n+1-m)} \widehat{\otimes} g^{(m)}\right)_{L^{2}(\mathbb{R})}, \quad n \in \mathbb{N}_{0},
$$

where the $L^{2}(\mathbb{R})$ "inner product" above is taken in the first variable of the tensor product. But the last expression is equal to

$$
\sum_{m=0}^{n+1}\left[(n+1-m)\left(h, f^{(n+1-m)}\right)_{L^{2}(\mathbb{R})} \widehat{\otimes} g^{(m)}+f^{(n+1-m)} \widehat{\otimes}\left(m\left(h, g^{(m)}\right)_{L^{2}(\mathbb{R})}\right)\right] .
$$

This on the other hand is the kernel of the $n$-th chaos of the right hand side of (3.2).

Now we discuss the adjoint (with respect to the dual pairing between $\mathcal{G}$ and $\mathcal{G}^{*}$ ) $D_{h}^{*}$ of $D_{h}$. 
Proposition 3.3. Let $h \in L^{2}(\mathbb{R}), \lambda^{\prime}, \lambda \in \mathbb{R}, \lambda^{\prime}<\lambda, \varphi \in \mathcal{G}_{\lambda}$. Then $D_{h}^{*} \varphi \in \mathcal{G}_{\lambda^{\prime}}$ and the following inequality holds

$$
\left\|D_{h}^{*} \varphi\right\|_{\lambda^{\prime}} \leq C_{\lambda^{\prime}, \lambda}^{*}|h|_{2}\|\varphi\|_{\lambda}
$$

where $C_{\lambda^{\prime}, \lambda}^{*}=\left(2\left(\lambda-\lambda^{\prime}\right)\right)^{-1 / 2} e^{(\lambda-1 / 2)}$.

Proof. Again the proof is straightforward by (2.3), an application of Schwarz' inequality and the formula

$$
g^{(n)}=h \widehat{\otimes} f^{(n-1)}, \quad n \in \mathbb{N}
$$

for the kernel of the $n$-th homogeneous chaos of $D_{h}^{*} \varphi$ (and of course $g^{(0)}=0$ ), the chaos of $\varphi$ being given by the sequence $\left\{f^{(n)}, n \in \mathbb{N}_{0}\right\}$.

Thus we have

Theorem 3.4. Let $h \in L^{2}(\mathbb{R})$ and $\lambda \in \mathbb{R}$. Then for all $\varepsilon>0, D_{h}^{*}$ acts continuously from $\mathcal{G}_{\lambda}$ into $\mathcal{G}_{\lambda-\varepsilon}$. In particular, $D_{h}^{*}$ is continuous on $\mathcal{G}$.

We remark in passing that - since $\mathcal{G}^{*} \subset(\mathcal{S})^{*}$ - the Hitsuda-Skorokhod integral (e.g., [HKP 93]) of processes in $\mathcal{G}^{*}$ indexed by an interval $I \subseteq \mathbb{R}$, say, is well-defined, and it gives an extension of the Itô integral with respect to Brownian motion. With a similar argument as in the proof of Proposition 3.3 it is then easy to show that the HitsudaSkorokhod integral is a continuous mapping from $L^{2}\left(I ; \mathcal{G}_{\lambda}\right)$ into $\mathcal{G}_{\lambda-\varepsilon}$ for all $\lambda \in \mathbb{R}, \varepsilon>0$.

Next we turn to translations in directions of $L^{2}(\mathbb{R})$. Again, let $h \in L^{2}(\mathbb{R})$, and define for $\varphi \in \mathcal{P}$

$$
\tau_{h} \varphi(\omega)=\varphi(\omega+h), \quad \omega \in \mathcal{S}^{\prime}(\mathbb{R})
$$

Proposition 3.5. Let $h \in L^{2}(\mathbb{R}), \varphi \in \mathcal{P}$, and $\lambda^{\prime} \in \mathbb{R}$. Set $\lambda_{0}:=\lambda^{\prime}+\ln \left(1+e^{-\lambda^{\prime}}|h|_{2}\right)$. Then for all $\lambda>\lambda_{0}$ the following estimate holds

$$
\left\|\tau_{h} \varphi\right\|_{\lambda^{\prime}} \leq\left(1-e^{-2\left(\lambda-\lambda_{0}\right)}\right)^{-1 / 2}\|\varphi\|_{\lambda}
$$

Proof. The following formula has been proved in [PY 92] (cf. also [HKP 93]):

$$
\varphi(\omega+h)=\sum_{n=0}^{\infty} \sum_{k=0}^{n}\left(\begin{array}{l}
n \\
k
\end{array}\right) I_{k}\left(f_{h}^{(n, n-k)}\right)
$$

where $\left\{f^{(n)}, n \in N_{0}\right\}$ is the sequence of integral kernels of the chaos expansion of $\varphi$, and $I_{n}\left(f^{(n)}\right)$ denotes the $n$-th multiple Wiener integral of $f^{(n)} \in L^{2}\left(\mathbb{R}^{n}\right)$. Moreover,

$$
f_{h}^{(n, n-k)}(v)=\int_{\mathbb{R}^{n-k}} f^{(n)}(v, u) h^{\otimes n-k}(u) d u, \quad v \in \mathbb{R}^{k} .
$$


Using the triangular and Schwarz' inequality we therefore get for any $\varepsilon>0$ the estimate

$$
\begin{aligned}
\left\|\tau_{h} \varphi\right\|_{\lambda^{\prime}} & \leq \sum_{n=0}^{\infty}\left|f^{(n)}\right|_{2} \sum_{k=0}^{n}\left(\begin{array}{l}
n \\
k
\end{array}\right) \sqrt{k !} e^{\lambda^{\prime} k}|h|_{2}^{n-k} \\
& \leq \sum_{n=0}^{\infty} \sqrt{n !}\left|f^{(n)}\right|_{2} e^{\lambda^{\prime} n}\left(1+e^{-\lambda^{\prime}}|h|_{2}\right)^{n} \\
& \leq\left(1-e^{-2 \varepsilon}\right)^{-1 / 2}\|\varphi\|_{\lambda_{0}+\varepsilon} .
\end{aligned}
$$

Clearly, Proposition 3.5 implies the following result.

Theorem 3.6. For every $h \in L^{2}(\mathbb{R}), \tau_{h}$ extends to a continuous operator on $\mathcal{G}$.

Finally, we turn to the Taylor series expansion of $\tau_{h} \varphi$.

Theorem 3.7. Let $h \in L^{2}(\mathbb{R}), \varphi \in \mathcal{G}$. Then

$$
\tau_{h} \varphi=\exp \left(D_{h}\right) \varphi
$$

where the exponential series

$$
\exp \left(D_{h}\right) \varphi=\sum_{n=0}^{\infty} \frac{1}{n !} D_{h}^{n} \varphi
$$

converges in $\mathcal{G}$.

Proof. Let $\varphi \in \mathcal{G}$ have chaos expansion given by the kernels $f^{(n)}, n \in N_{0}$, and denote by $\varphi^{(n)}$ the $n$-th multiple Wiener integral of $f^{(n)}$. Iteration of the formula for $D_{h} \varphi$ mentioned in the proof of Proposition 3.1 gives for all $M \in \mathbb{N}, n \in \mathbb{N}_{0}$,

$$
\sum_{m=0}^{M} \frac{1}{m !} D_{h}^{m} \varphi^{(n)}=\sum_{m=0}^{M}\left(\begin{array}{l}
n \\
m
\end{array}\right) I_{n-m}\left(f_{h}^{(n, m)}\right)
$$

where we used the same notation as in the proof of Proposition 3.5, and we make the convention $\left(\begin{array}{c}n \\ m\end{array}\right)=f_{h}^{(n, n-m)}=0$ for $m>n$. Upon comparison with the formula for $\tau_{h} \varphi$ given in the proof of Proposition 3.5, we find

$$
\tau_{h} \varphi-\sum_{m=0}^{M} \frac{1}{m !} D_{h}^{m} \varphi=\sum_{n=M+1}^{\infty} \sum_{m=M}^{n}\left(\begin{array}{l}
n \\
m
\end{array}\right) I_{n-m}\left(f_{h}^{(n, m)}\right) .
$$

Thus by the triangular and Schwarz' inequality we obtain

$$
\left\|\tau_{h} \varphi-\sum_{m=0}^{M} \frac{1}{m !} D_{h}^{m} \varphi\right\|_{\lambda} \leq \sum_{n=M+1}^{\infty}\left|f^{(n)}\right|_{2} \sum_{m=0}^{n}\left(\begin{array}{c}
n \\
m
\end{array}\right) \sqrt{m !}|h|_{2}^{n-m} e^{\lambda m} .
$$

The last term is the remainder of a series which in the proof of Proposition 3.5 has been shown to be convergent. Thus the exponential series $\exp \left(D_{h}\right) \varphi$ converges for all $\lambda \in \mathbb{R}$ in $\mathcal{G}_{\lambda}$ to $\tau_{h} \varphi$. This concludes the proof of the theorem. 


\section{A Criterium in Terms of the $\mathcal{S}$-Transform}

In this section we prove our main result and discuss some of its consequences. First we define the $\mathcal{S}$-transform of the elements of $\mathcal{G}^{*}$.

Let $\lambda \in \mathbb{R}$ and consider $\Phi \in \mathcal{G}_{\lambda}$. Define its $\mathcal{S}$-transform $\mathcal{S} \Phi$ by

$$
\mathcal{S} \Phi(\xi):=<\bar{\Phi},: e^{X_{\xi}}:>, \xi \in \mathcal{S}(\mathbb{R})
$$

where $\langle\cdot, \cdot\rangle$ denotes the canonical dual pairing between $\mathcal{G}^{*}$ and $\mathcal{G}$. Note that $: e^{X_{\xi}}: \in \mathcal{G}$ (which is proved as in Example 3.1) so that for all $\Phi \in \mathcal{G}^{*}, S \Phi$ is an everywhere defined function on $\mathcal{S}(\mathbb{R})$. Moreover, if $\lambda \geq 0, \varphi \in \mathcal{G}_{\lambda}$, then

$$
\mathcal{S} \varphi(\xi)=\int \varphi(\omega+\xi) d \mu(\omega) .
$$

Also, it is easy to see that $\mathcal{S} \Phi(\xi), \xi \in \mathcal{S}(\mathbb{R})$ has an extension for $\xi \in \mathcal{S}_{\mathbb{C}}(\mathbb{R})$. Indeed, if for $\xi \in \mathcal{S}_{\mathbb{C}}(\mathbb{R})$ the canonical coordinate process $X_{\xi}$ is defined by $X_{\xi}:=X_{\operatorname{Re} \xi}+i X_{\mathbb{I m} \xi}$, this extension is also given by

$$
\begin{gathered}
\mathcal{S} \Phi(\xi):=<\bar{\Phi},: e^{X_{\xi}}:>, \xi \in \mathcal{S}_{\mathbb{C}}(\mathbb{R}), \\
: e^{X_{\xi}}:(\omega)=e^{<\omega, \xi>-\frac{1}{2}(\bar{\xi}, \xi)}, \quad \omega \in \mathcal{S}^{\prime}(\mathbb{R}),
\end{gathered}
$$

where now

and the last inner product is the one of $L_{\mathbb{C}}^{2}(\mathbb{R})$. Moreover, this extension is G-entire everywhere in $\mathcal{S}_{\mathbb{C}}(\mathbb{R})$, i.e., for all $\xi, \eta \in \mathcal{S}_{\mathbb{C}}(\mathbb{R})$, the mapping $z \mapsto S \Phi(\xi+\lambda \eta) ; z \in \mathbb{C}$ is entire analytic.

Consider a complex-valued function $F$ on $\mathcal{S}_{\mathbb{C}}(\mathbb{R})$ which is $\mathrm{G}$-entire. We are interested in a criterium on $F$ which allows to conclude that it is the $S$-transform of an element in $\mathcal{G}$ or $\mathcal{G}^{*}$. Such results have been shown for $(\mathcal{S})$ and $(\mathcal{S})^{*}$ in [Ko 80, KPS 91, Le 91, MY 91, PS 91] and other papers, and in the context of Hida distributions they seem to be useful for applications.

Theorem 4.1. Assume that there exists a bounded, non-negative quadratic form $Q$ on $L^{2}(\mathbb{R})$ which is in the trace class, and such that for all $\xi \in \mathcal{S}(\mathbb{R}), z \in \mathbb{C}$,

$$
|F(z \xi)| \leq K e^{|z|^{2} Q(\xi, \xi)}
$$

for some positive constant $K$. Let $\rho(\lambda):=2 e^{2(\lambda+1)} \operatorname{Tr} Q, \lambda \in \mathbb{R}$. If $\rho(\lambda)<1$, then there exists a unique $\Phi \in \mathcal{G}_{\lambda}$ so that $S \Phi=F$. Moreover

$$
\|\Phi\|_{\lambda} \leq K(2 \pi)^{-\frac{1}{4}}(1-\rho(\lambda))^{-\frac{1}{2}} .
$$


Corollary 4.2. Assume that (4.1) holds. Then there exists a unique $\Phi \in \mathcal{G}^{*}$ so that $S \Phi=F$.

Proof. Choose $\lambda<0,|\lambda|$ large enough so that $\rho(\lambda)<1$.

Corollary 4.3. Suppose that for every $\varepsilon>0$ there exists $K_{\varepsilon}>0$ so that for all $\xi \in \mathcal{S}(\mathbb{R}), z \in \mathbb{C}$,

$$
|F(z \xi)| \leq K_{\varepsilon} e^{\varepsilon|z|^{2} Q(\xi, \xi)} .
$$

Then there exists a unique $\varphi \in \mathcal{G}$ so that $S \varphi=F$.

Proof. Replacing $Q$ in $\rho(\lambda)$ by $\varepsilon Q$ we get $\rho(\lambda)=2 \varepsilon e^{2(\lambda+1)} \operatorname{Tr} Q$. For every $\lambda \in \mathbb{R}$, choose $\varepsilon$ small enough so that $\rho(\lambda)<1$. Then $S^{-1} F$ belongs to $\mathcal{G}_{\lambda}$ for all $\lambda \in \mathbb{R}$, i.e., it belongs to $\mathcal{G}$.

Corollary 4.4. Assume that $\left\{F_{n}, n \in \mathbb{N}\right\}$ is a sequence of functions on $\mathcal{S}(\mathbb{R})$ which have everywhere ray entire extensions. Suppose that $\left\{F_{n}, n \in \mathbb{N}\right\}$ is pointwise Cauchy, that the estimate (4.1) holds for every $F_{n}, n \in \mathbb{N}$, uniformly in $n$, and that $\lambda \in \mathbb{R}$ is such that $\rho(\lambda)<1$. Then there exists a unique element $\Phi \in \mathcal{G}_{\lambda}$, so that $\Phi_{n}:=S^{-1} F_{n}$ converges weakly to $\Phi$ in $\mathcal{G}_{\lambda}$.

Proof. The complex algebra $\mathcal{E} \subset \mathcal{G}$ generated by the functions $\omega \mapsto \exp \langle\omega, \xi\rangle, \xi \in$ $\mathcal{S}(\mathbb{R}), \omega^{\prime} \in \mathcal{S}^{\prime}(\mathbb{R})$, is dense in $\mathcal{G}_{\lambda}, \lambda \in \mathbb{R}$. The hypothesis implies that $\Phi_{n}$ is Cauchy on the dense set $\mathcal{E}$ in $\mathcal{G}_{\lambda}$. The uniform bound together with inequality (4.2) show that $\left\{\Phi_{n}, n \in \mathbb{N}\right\}$ is bounded in $\mathcal{G}_{\lambda}$. Therefore $\left\{\Phi_{n}, n \in \mathbb{N}\right\}$ is weakly Cauchy in $\mathcal{G}_{\lambda}$. Since $\mathcal{G}_{\lambda}$ is a Hilbert space, it is weakly complete, and therefore there exists a unique $\Phi \in \mathcal{G}_{\lambda}$ so that $\left\{\Phi_{n}, n \in \mathbb{N}\right\}$ converges weakly to $\Phi$.

Remarks. 1. By general theory (e.g., [Ka 76]) we can replace everywhere above $Q(\xi, \xi)$ by $\|A \xi\|_{L^{2}(\mathbb{R})}^{2}$, where $A$ is a symmetric Hilbert-Schmidt operator on $L^{2}(\mathbb{R})$.

2. We have tried to construct an example of an element in $\mathcal{G}_{\lambda}$ with an $\mathcal{S}$-transform which does not admit a bound of the type (4.1), however without success. Therefore, the "goodness" of the criterium given in Theorem 4.1 remains an open problem.

We now prove Theorem 4.1 by following the arguments in [PS 91] quite closely (cf. also [HKP 93, Chapter 4]).

Proof of Theorem 4.1. Let $\xi \in \mathcal{S}(\mathbb{R})$, and consider the entire analytic function $z \mapsto F(z \xi)$. Its power series expansion at the origin reads

$$
F(z \xi)=\sum_{n=0}^{\infty} z^{n} F^{(n)}(\xi) .
$$

Clearly we have

$$
F^{(n)}(\xi)=\left.\frac{1}{n !} \frac{d^{n}}{d z^{n}} F(z \xi)\right|_{z=0}
$$

and it is easy to see that

$$
F^{(n)}(\xi)=\frac{1}{n !}\left(D_{\xi}^{n} F\right)(0)
$$


where $D_{\xi}$ denotes the Gâteaux-derivative in direction $\xi$. The last relation shows that $F^{(n)}$ is homogeneous in $\xi$ of degree $n$.

Assume for the moment that $Q(\xi, \xi) \neq 0$, and normalize $\xi$ so that $Q(\xi, \xi)=1$. Then Cauchy's theorem entails the estimate

$$
\left|F^{(n)}(\xi)\right| \leq K R^{-n} e^{R^{2}}
$$

for every $R>0$, and optimizing we find

$$
\left|F^{(n)}(\xi)\right| \leq K n^{-\frac{n}{2}}(2 e)^{\frac{n}{2}}
$$

Using the homogeneity of $F^{(n)}$ we therefore get for arbitrary $\xi \in \mathcal{S}(\mathbb{R})$ with $Q(\xi, \xi) \neq 0$ the estimate

$$
\left|F^{(n)}(\xi)\right| \leq K n^{-\frac{n}{2}}(2 e)^{\frac{n}{2}} Q(\xi, \xi)^{\frac{n}{2}} .
$$

If $Q(\xi, \xi)=0$, Cauchy's theorem shows that $F^{(n)}(\xi)=0$, and therefore the bound (4.4) holds for every $\xi \in \mathcal{S}(\mathbb{R})$.

Now define the following symmetric $n$-multilinear form $f^{(n)}$ on $\mathcal{S}(\mathbb{R})$ (cf. [KL 93]). For $\xi_{1}, \ldots, \xi_{n} \in \mathcal{S}(\mathbb{R})$ set

$$
f^{(n)}\left(\xi_{1}, \ldots, \xi_{n}\right):=\frac{1}{n !}\left(D_{\xi_{1}} \cdots D_{\xi_{n}} F\right)(0)
$$

Then $f^{(n)}$ can be obtained from $F^{(n)}$ by polarization:

$$
f^{(n)}\left(\xi_{1}, \ldots, \xi_{n}\right)=\frac{1}{2^{n} n !} \sum_{\varepsilon} \varepsilon_{1} \cdots \varepsilon_{n} F^{(n)}\left(\varepsilon_{1} \xi_{1}+\ldots+\varepsilon_{n} \xi_{n}\right)
$$

where the sum $\sum_{\varepsilon}$ is over all $n$-tuples $\varepsilon=\left(\varepsilon_{1}, \ldots, \varepsilon_{n}\right)$ with $\varepsilon_{i}= \pm 1, i=1, \ldots, n$. In order to obtain a bound for $f^{(n)}$, assume again first that $Q\left(\xi_{i}, \xi_{i}\right) \neq 0$, and by normalization $Q\left(\xi_{i}, \xi_{i}\right)=1$ for all $i=1,2, \ldots, n$. Then by $(4.4)$

$$
\begin{aligned}
\left|f^{(n)}\left(\xi_{1}, \ldots, \xi_{n}\right)\right| & \leq K \frac{1}{2^{n} n !} n^{-n / 2}(2 e)^{n / 2} \sum_{\epsilon} Q\left(\varepsilon_{1} \xi_{1}+\ldots+\varepsilon_{n} \xi_{n}, \varepsilon_{1} \xi_{1}+\ldots+\varepsilon_{n} \xi_{n}\right)^{n / 2} \\
& \leq K \frac{1}{2^{n} n !} n^{-n / 2}(2 e)^{n / 2} 2^{n} n^{n} \\
& =K \frac{1}{n !} n^{n / 2}(2 e)^{n / 2}
\end{aligned}
$$

where we used the triangular inequality for $Q^{\frac{1}{2}}$ in the second step. By multi-linearity of $f^{(n)}$ we have

$$
\left|f^{(n)}\left(\xi_{1}, \ldots, \xi_{n}\right)\right| \leq K \frac{1}{n !}(2 e n)^{\frac{n}{2}} \prod_{i=1}^{n} Q\left(\xi_{i}, \xi_{i}\right)^{\frac{1}{2}}
$$


if $Q\left(\xi_{i}, \xi_{i}\right) \neq 0$ for all $i$. If there exists $i$ so that $Q\left(\xi_{i}, \xi_{i}\right)$, then we can show as above that $\left(D_{\xi_{i}} F\right)(0)=0$ and therefore also $f^{(n)}\left(\xi_{1}, \ldots, \xi_{n}\right)=0$. Thus also in this case the inequality (4.5) holds true. Now use Stirling's theorem to obtain

$$
\left|f^{(n)}\left(\xi_{1}, \ldots, \xi_{n}\right)\right| \leq K \cdot(2 \pi)^{-\frac{1}{4}} \frac{1}{\sqrt{n !}}\left(2 e^{2}\right)^{\frac{n}{2}} \prod_{i=1}^{n} Q\left(\xi_{i}, \xi_{i}\right)^{\frac{1}{2}}
$$

Let $\left\{e_{k}, k \in \mathbb{N}\right\}$ be a CONS of $L^{2}(\mathbb{R})$. Then we have

$$
\begin{aligned}
\left\|f^{(n)}\right\|_{\mathrm{H} . S .}^{2 .} & =\sum_{k_{1}, \ldots, k_{n}=1}^{\infty}\left|f^{(n)}\left(e_{k_{1}}, \ldots, e_{k_{n}}\right)\right|^{2} \\
& \leq K^{2}(2 \pi)^{-\frac{1}{2}} \frac{1}{n !}\left(2 e^{2}\right)^{n} \cdot \sum_{k_{1}, \ldots, k_{n}=1}^{\infty} Q\left(e_{k_{1}}, e_{k_{1}}\right) \cdots Q\left(e_{k_{n}}, e_{k_{n}}\right) \\
& =K^{2}(2 \pi)^{-\frac{1}{2}} \frac{1}{n !}\left(2 e^{2} \operatorname{Tr} Q\right)^{n} \\
& =K^{2}(2 \pi)^{-\frac{1}{2}} \frac{1}{n !} e^{-2 \lambda n} \rho(\lambda)^{n}
\end{aligned}
$$

Since $f^{(n)}$ has a finite Hilbert-Schmidt norm, it may be identified with a symmetric element in $L^{2}\left(\mathbb{R}^{n}\right)$, and its $L^{2}\left(\mathbb{R}^{n}\right)$-norm is equal to the above Hilbert-Schmidt norm. Thus we may set

$$
\Phi:=\sum_{n=0}^{\infty} I_{n}\left(\overline{f^{(n)}}\right)
$$

and we have

$$
\begin{aligned}
\|\Phi\|_{\lambda}^{2} & =\sum_{n=0}^{\infty} n ! e^{2 \lambda n}\left\|f^{(n)}\right\|_{L^{2}\left(\mathbb{R}^{n}\right)}^{2} \\
& \leq K^{2}(2 \pi)^{-\frac{1}{2}} \frac{1}{1-\rho(\lambda)} .
\end{aligned}
$$

Hence $\Phi \in \mathcal{G}_{\lambda}$. It is straightforward to compute the $S$-transform of $\Phi$ (cf. also [HKP 93, Chapt. 2]):

$$
\begin{aligned}
S \Phi(\xi) & =\sum_{n=0} f^{(n)}(\xi, \ldots, \xi) \\
& =\sum_{n=0} F^{(n)}(\xi) \\
& =F(\xi) .
\end{aligned}
$$

Also, by inspection the above arguments show that $\Phi$ is uniquely determined by $F$. 
Example 4.5. We reconsider Example 2.1. It is trivial to compute the $\mathcal{S}$-transform of $Y(t)=\exp \left(B(t)-\frac{1}{2} t\right): S Y(t)(\xi)=\exp \left(\int_{0}^{t} \xi(s) d s\right)$. Consider

$$
\exp \left(\int_{0}^{t} \eta(s) d s+z \int_{0}^{t} \xi(s) d s\right), \quad \eta, \xi \in \mathcal{S}(\mathbb{R}), z \in \mathbb{C}
$$

which is obviously the everywhere ray-entire extension of $\mathcal{S} Y(t)$. Now choose

$$
Q(\xi, \xi)=\left(\int_{0}^{t} \xi(s) d s\right)^{2}
$$

which is clearly a quadratic form on $\mathcal{S}(\mathbb{R})$, and it is trace class on $L^{2}(\mathbb{R})$ (with $\left.\operatorname{Tr} Q=t\right)$. It is trivial to verify the bound (4.3) for any $\varepsilon>0\left(\right.$ choose $\left.K_{\varepsilon}=\exp \left((4 \varepsilon)^{-1}\right)\right)$. Thus, $\boldsymbol{Y}(\boldsymbol{t}) \in \mathcal{G}$.

Example 4.6. Consider again Example 2.2, Donsker's delta function $\delta_{x} \circ B(t), t \geq 0$. Its $\mathcal{S}$-transform is given by (e.g., [HKP 93])

$$
(2 \pi t)^{-1 / 2} \exp \left(-\frac{1}{2 t}\left(x-\int_{0}^{t} \xi(s) d s\right)^{2}\right) .
$$

Again it is easy to see that this function on $\mathcal{S}(\mathbb{R})$ has everywhere a ray-analytic extension (just replace above $\xi$ by $\eta+z \xi$ ), and that with the same $Q$ as in the previous example the bound (4.1) holds. Thus we have $\delta_{x} \circ B(t) \in \mathcal{G}^{*}$.

\section{References.}

[CLP 93] Cochran, G., Lee, J.-S. and Potthoff, J.: Stochastic Volterra equations with singular kernels; Preprint (1993)

[CP 92] Cochran, G. and Potthoff, J.: Fixed point principles for stopchastic partial differential equations; Preprint (1992), to appear in: Proc. Int. Conf. Dynamics of Complex and Irrgeular Systems

[FPS 91] de Faria, M., Potthoff, J. and Streit, L.: The Feynman integrand as a Hida distribution; J. Math. Phys. 32 (1991) 2123-2127

[FP 89] : Feyel, D. and de la Pradelle, A.: Espaces de Sobolev Gaussiens; Ann. Inst. Fourier 39 (1989) 87.5-908

[GV 68] Gel'fand, IM. and Vilenkin, N.Ya.: Generalized Functions IV. New York, London: Academic Press (1968)

[HKP 93] Hida, T., Kuo, H.-H., Potthoff, J. and Streit, L.: White Noise - An Infinite Dimensional Calculus. Dordrecht: Kluwer (1993)

[HPS 88] Hida, T., Potthoff, J. and Streit, L.: Dirichlet forms and white noise analysis; Commun. Math. Phys. 116 (1988) 235-245

[HP 57] Hille, E. and Phillips, R.S.: Functional Analysis and Semigroups. Providence: American Mathematical Society (1957)

[Ka 76] Kato, T.: Periurbation Theory for Linear Operators. Berlin, Heidelberg, New York: Springer (1976)

[KL 93] Kondratiev, Y., Leukert, P., Potthoff, J., Streit, L. and Westerkamp, W.: Generalized functionals in Gaussian spaces - the characterization theorem revised; Preprint (1993) 
[KS 92] Khandekar, D.C. and Streit, L.: Constructing the Feynman integrand; Ann. Physik 1 (1992) 49-55

[Ko 80] Kondratiev, Y.: Nuclear spaces of entire functions in problems of infinite-dimensional analysis; Soviet Math. Dokl. 22 (1980) 588-592

[KP 90] Kuo, H.-H. and Potthoff, J.: Anticipating stochastic integrals and stochastic differential equations; in: White Noise Analysis - Mathematics and Applications, T. Hida, H.-H. Kuo, J. Potthoff and L. Streit (eds.). Singapore: World Scientific (1990)

[KPS 91] Kuo, H.-H., Potthoff, J. and Streit, L.: A characterization of white hoise test functionals; Nagoya Math. J. 121 (1991) 185-194

[LLS 93] Laschek, A., Leukert, P., Streit, L. and Westerkamp, W.: Quantum mechanical propagators in terms of Hida distributions; Preprint (1993)

[Le 91] Lee, Y.-J.: Analytic version of test functionals, Fourier transform and a characterization of measures in white noise calculus; J. Funct. Anal. 100 (1991) $359-380$

[LM 88] Lindsay, M. and Massen, H.: An integral kernel approach to noise; in: Quantum Probability and Applications II, L. Accardi and W. von Waldenfels (ed.'s). Berlin, Heidelberg, New York: Springer (1988)

[LOU 91] Lindstrøm, T., Øksendal, B. and Ubøe, J.: Wick multiplication and Itô-Skorohod stochastic differential equations; Preprint (1991), to appear in: Ideas and Methods in Mathematical Physics

[Me 83] Meyer, P.A.: Quelques résultats analytiques sur le semigroupe d'Ornstein-Uhlenbeck en dimension infinie; in Theory and Application of Random Fields; G. Kallianpur (ed.). Berlin, Heidelberg, New York: Springer (1983)

[MY 87] Meyer, P.A. and Yan, J.-A.: A propos des distributions sur l'espace de Wiener; Séminaire de Probabilités XXI; J. Azéma and M. Yor (ed.s). Berlin, Heidelberg, New York: Springer (1987)

[MY 91] Meyer, P.A. and Yan, J.-A.: Les "fonctions caractéristiques" des distributions sur l'espace de Wiener; Séminaire de Probabilités XXV; J. Azéma, P.A. Meyer and M. Yor (ed.s). Berlin, Heidelberg, New York: Springer (1991)

[Ne 73] Nelson, E.: Probability theory and Euclidean quantum field theory; in Constructive Quantum Field Theory, G. Velo and A. Wightman (ed.s). Berlin, Heidelberg, New York: Springer (1973)

[Po 92] Potthoff, J.: White noise methods for stochastic partial differential equations; in: Stochastic Partial Differential Equations and Their Applications, B.L. Rozovskii, R.B. Sowers (ed.'s). Berlin, Heidelberg, New York: Springer (1992)

[Po 93] Potthoff, J.: White noise approach to parabolic stochastic partial differential equations; in preparation

[PS 93a] Potthoff, J. and Sundar, P.: Law of large numbers and central limit theorem for Donsker's delta function; Stochastics and Stochastics Reports 42 (1993) 135-150

[PS 93b] Potthoff, J. and Sundar, P.: Law of large numbers and central limit theorem for Donsker's delta function of diffusions I; Preprint (1993)

[PS 91] Potthoff, J. and Streit, L.: A characterization of Hida distributions; J. Funct. Anal. 101 (1991) 212-229

[PS 93] Potthoff, J. and Streit, L.: Invariant states on random and quantum fields: $\phi-$ bounds and white noise analysis; J. Funct. Anal. 111 (1993) 295-311

[PY 92] Potthoff, J. and Yan J.A.: Some results about test and generalized functionals of white noise; in: Probability Theory, L.H.Y. Chen et al. (ed.'s). Berlin, New York: de Gruyter (1992)

[Si 74] Simon, B.: The $P(\Phi)_{2}$ Euclidean (Quantum) Field Theory. Princeton: Princeton University Press (1974)

[Su 85] Sugita, H.: Sobolev spaces of Wiener functionals and Malliavin's calculus; $J$. Math. Kyoto Univ. 25 (1985) 31-48 
[ÜZ 93] Üstünel, A.S. and Zakai, M.: The composition of Wiener functionals with non absolutely continuous shifts; Preprint (1993)

[Wa 83] Watanabe, S.: Malliavin's calculus in terms of generalized Wiener functionals; in Theory and Application of Random Fields, G. Kallianpur (ed.). Berlin, Heidelberg, New York: Springer (1983)

[Wa 84]: Watanabe, S.: Stochastic Differential Equations and Malliavin Calculus. Bombay: Tata Inst. of Fundamental Research (1984) 